\title{
EXCITON THEORY
}

The application of the Exciton theory provides structural information of the aggregates regarding its simplest geometry model (e.g. the chromophore spacing $R$, and the relative spatial arrangement of the transition dipole moments of the molecules forming the aggregates $\alpha$, in Figure

$2 \mathrm{~b}$ of manuscript) ${ }^{1,2,3}$. Thus, the $\alpha$ angle between the monomer units in the aggregate can be calculated (equation 1, Table 1) from the ratio between the areas of the long-wavelength $\left(\mathrm{A}_{1}\right)$ and the short-wavelength $\left(A_{2}\right)$ excitation bands (Figure 1, Table 1). The interaction energy $\left(U\right.$, in $\left.\mathrm{cm}^{-1}\right)$ between the monomers forming the aggregate is calculated (equation 2) from the long-wavenumber $\left(v_{2}\right.$, in $\left.\mathrm{cm}^{-1}\right)$ and the short-wavenumber $\left(v_{1}\right.$, in $\left.\mathrm{cm}^{-1}\right)$ excitation bands (Figure 1, Table 1). The ratio between the areas of the long-wavelength and the short-wavelength excitation bands as well as the wavelengths values are experimentally calculated as described elsewhere ${ }^{3}$.

$$
\begin{aligned}
& \tan ^{2}(\alpha / 2)=\mathrm{A}_{1} / \mathrm{A}_{2} \\
& U=\left(v_{2}-v_{1}\right) / 2
\end{aligned}
$$

For oblique configuration, the separation distance between the molecules ( $R$, in $\AA$ ) can be obtained from equation 3 , where $v_{\mathrm{m}}\left(\mathrm{cm}^{-1}\right)$ and $\int \varepsilon(v) \mathrm{d} v\left(\mathrm{M}^{-1} \mathrm{~cm}^{-1}\right)$ are the wavenumber at the maximum intensity and the area of the absorption band of RBG $1,{ }^{4}$ respectively (see Figure 2, Table 1).

$$
R=\left(\frac{1.85 \cdot 10^{2} \cdot \int \varepsilon(v) d v \cdot[\cos (\alpha)+3 \cdot \sin (\alpha / 2)]}{U \cdot v_{m}}\right)^{1 / 3}
$$

(1) López-Arbeloa, F.; Martínez-Martínez, V.; Bañuelos-Prieto, J.; López-Arbeloa, I. Langmuir 2002, 18, 2658, and references there in.

(2) Bojarski, P.; Matczuk, A.; Bojarski, C.; Kawski, A.; Kuklinski, B.; Zurkowska, G.; Diehl, H. Chem. Phys. 1996, 210, 485.

(3) Ferrer, M. L.; del Monte, F. Langmuir 2003, 19, 2782, and references there in.

(4) The extinction coefficient of RBG1 was measured in transmittance mode on a bulk sample of $3 \mathrm{~mm}$ thickness using a Varian 2300 Spectrophotometer. 


\section{FIGURES:}

Figure S1: Bands resulting from RBG1 excitation spectrum subtraction to RBG4 excitation spectrum. Every excitation spectra is normalized prior to subtraction.

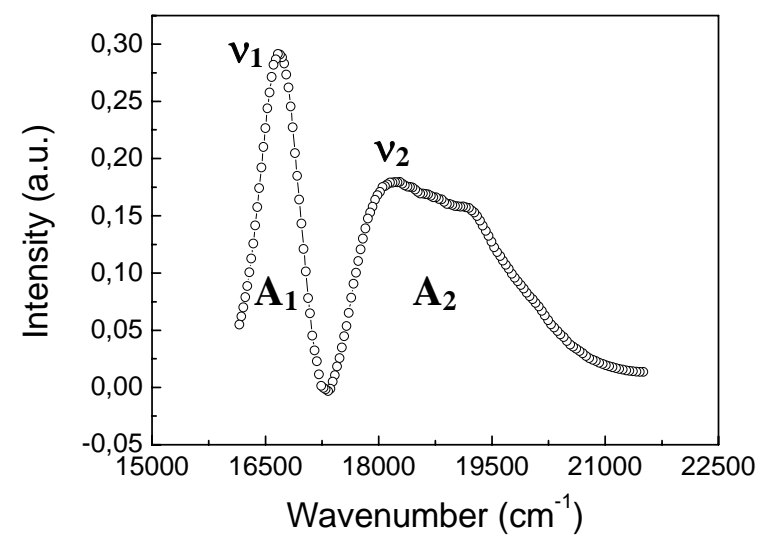

Figure S2: Plot of the RBG1 extinction coefficient versus the wavenumber $\left(\int \varepsilon(v) d v\right.$ $\left.=3.4 \times 10^{7} \mathrm{M}^{-1} \mathrm{~cm}^{-1}, v_{\mathrm{m}}=17825 \mathrm{~cm}^{-1}\right)$.

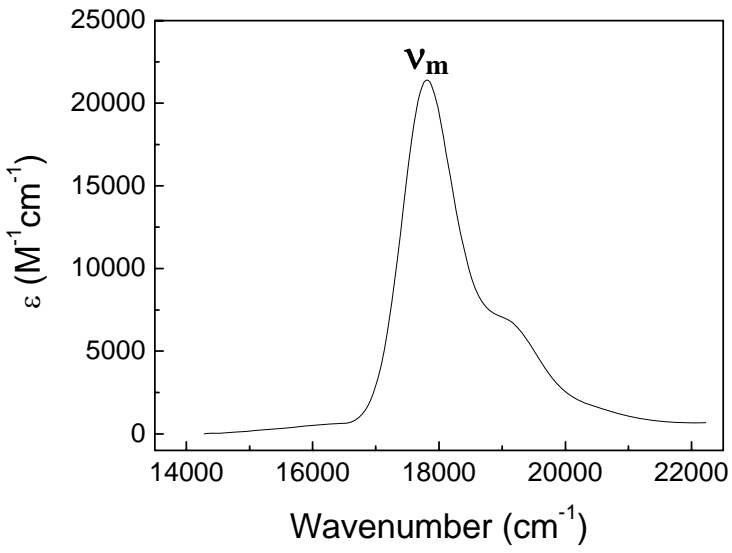

Table S1: Data $\left(\mathbf{A}_{1} / \mathbf{A}_{2}\right.$ and $\left.\boldsymbol{U}\right)$ obtained from bands resulting from RBG1 excitation spectrum subtraction to RBG3, RBG4, RBG5, RBG6 and RBG7, respectively. Values of $\boldsymbol{\alpha}$ and $\boldsymbol{R}$ calculated from such data.

\begin{tabular}{|c|c|c|c|c|}
\hline $\mathbf{R B G}$ & $\mathbf{A}_{\mathbf{1}} / \mathbf{A}_{\mathbf{2}}$ & $\boldsymbol{U}\left(\mathbf{c m}^{\mathbf{- 1}}\right)$ & $\boldsymbol{\alpha} \mathbf{(}^{\mathbf{9}}$ & $\boldsymbol{R}(\boldsymbol{\AA})$ \\
\hline $\mathbf{3}$ & 0.93 & 544 & 88 & 11.1 \\
\hline $\mathbf{4}$ & 0.59 & 760 & 75 & 9.8 \\
\hline $\mathbf{5}$ & 0.56 & 774 & 74 & 9.8 \\
\hline $\mathbf{6}$ & 0.59 & 801 & 75 & 9.7 \\
\hline $\mathbf{7}$ & 0.55 & 832 & 73 & 9.6 \\
\hline
\end{tabular}


We can also apply the Exciton theory to the experimentally measured lifetime values $(\tau)$ of RBG2-RBG7, ${ }^{5,6,7}$ and thus obtain the $\theta$ angle values complementary to $\alpha$ (i.e., $\alpha+2 \theta=180^{\circ}$ ). Thus, the radiative rate constant of fluorescent aggregates can be correlated to the radiative rate constant of the monomer unit and the $\theta$ angle characteristic of aggregates in oblique configuration (4):

$$
\mathrm{kr}_{\mathrm{D}}=\mathrm{kr}_{\mathrm{M}} \cos ^{2} \theta
$$

where $\mathrm{kr}_{\mathrm{D}}$ is the radiative rate constant of the fluorescent dimers and $\mathrm{kr}_{\mathrm{M}}$ is the radiative rate constant of the monomer unit. The radiative rate constant of the monomer and the fluorescent aggregates were directly calculated from the lifetime $(\tau)$, which is a valid approximation taking into account the high fluorescent quantum yield calculated for RBG1 $(\phi=1) .{ }^{8,9}$

Table S2

\begin{tabular}{|c|c|c|}
\hline & $\tau$ (ns) & $\left.\boldsymbol{\theta} \mathbf{(}^{\mathbf{0}}\right)$ \\
\hline RBG1 & $4.0 \pm 0.2$ & -- \\
\hline RBG2 & $4.1 \pm 0.2$ & -- \\
\hline RBG3 & $4.4 \pm 0.2$ & 47.6 \\
\hline RBG4 & $5.3 \pm 0.2$ & 52.1 \\
\hline RBG5 & $5.5 \pm 0.2$ & 52.9 \\
\hline RBG6 & $5.3 \pm 0.2$ & 52.1 \\
\hline RBG7 & $5.4 \pm 0.2$ & 52.5 \\
\hline
\end{tabular}

(5) McRae, E. G.; Kasha, M. J. Chem. Phys. 1961, 11, 38.

(6) Kasha, M. Rev. Mod. Phys. 1959, 31, 162.

(7) McRae, E. G.; Kasha, M. Physical Processes in Radiation Biology, 1st ed.; Academic Press: New York, 1964.

(8) Itho, K.; Honda, K. Chem. Phys. Lett. 1982, 87, 213.

(9) Vogel, M.; Rettig, W.; Sens, R.; Drexhage, K. H. Chem. Phys. Lett. 1988, 147, 461. 
Scheme I: Proposed mechanism of RB dimers formation in a pure silica matrix based on fluorescent characterization (e.g., excitation and emission spectroscopy, fluorescence lifetimes and fluorescence anisotropy). At low concentrations (RBS1), single RB molecules are adsorbed on the porous surface but not totally constrained by the pore cage. The progressive adsorption of molecules on the porous surface cause the increase of the adsorption angle $(\phi)$ that gives rise to a rearrangement of the adsorbed species with the subsequent change of the fluorescent properties; e.g. from fluorescent J-dimers in an in-plane oblique angle configuration $\left(\phi<54.7^{\circ}\right.$, RBS2-RBS3) to non-fluorescent H-dimers in an in-plane oblique angle configuration and ultimately, in a parallel plane twist angle configuration ( $\phi>54.7^{\circ}$, RBS4-RBS7) (see reference 3 for further details).
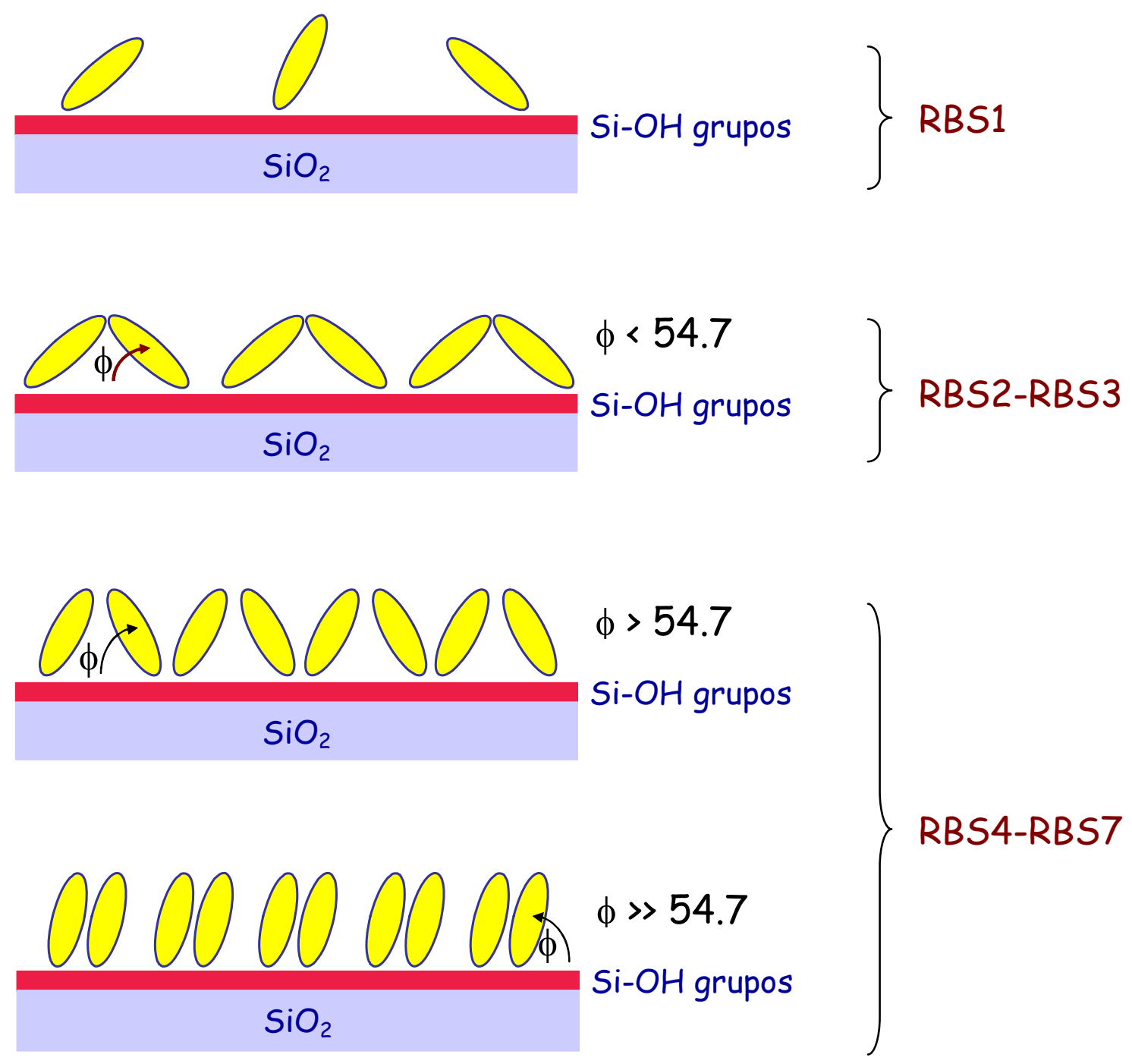
Figure 3: Fluorescence intensity of $\mathrm{RB}$ and NR versus the square root of dye concentration, in hybrid (RBG and NRG) and pure silica (RBS) sol-gel materials. The decrease in fluorescence intensity observed in pure silica matrixes when RB concentration increases above $10^{-4} \mathrm{M}$ (RBS3), is a general trend for different xanthene type molecules (e.g., R110, R101 and R19). ${ }^{3}$

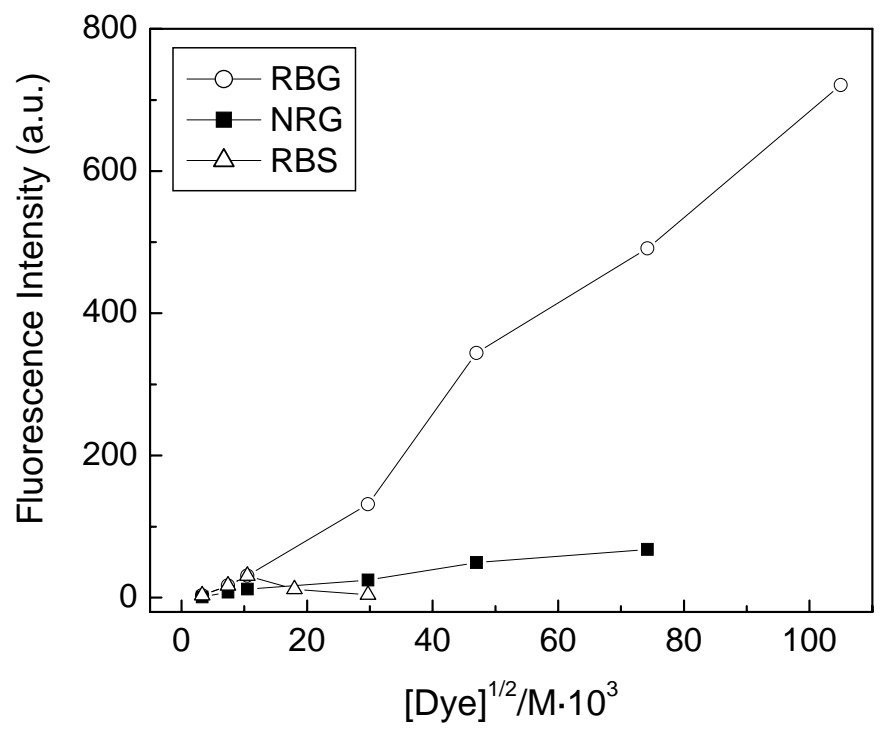

